\title{
Resonant Spin Splitting in III-V Semiconductors
}

\section{A. SKIERKOWSKI* AND J.A. MAJEWSKI}

Institute of Theoretical Physics, Warsaw University

Hoża 69, 00-681 Warszawa, Poland

\begin{abstract}
We present first-principles studies of the zero field spin splitting of energy bands in typical III-V semiconductors. Our calculations reveal that the strain induces linear- $\boldsymbol{k}$ spin splitting of the conduction band in the $\Gamma$ point, which is linear in strain, and determine the magnitude of the so-called acoustic phonon constant that characterizes the magnitude of the spin splitting. In addition, we show that optical phonons lead to spin-flip processes and we present quantitative results for the spin-phonon deformation potentials in GaAs. Most importantly, the calculations show that the linear- $\boldsymbol{k}$ spin splitting can be resonantly enhanced when bands cross in a particular point of the Brillouin zone. This resonant enhancement of the bulk inversion asymmetry coupling constant by more than one order of magnitude was observed in both valence and conduction bands and can be steered by the application of the external stress. This allows tailoring of the spin relaxation and spin precession of conduction electrons in nanostructures to a much larger extent than was hitherto assumed.
\end{abstract}

PACS numbers: 31.15.Ar, 71.70.Ej, 71.20.-b, 71.20.Nr

\section{Introduction}

The present surge in interest for spin devices and spin relaxation effects calls for a detailed understanding of the spin dynamics in semiconductor nanostructures $[1,2]$. The spin splitting of the bands caused by the spin-orbit coupling induces a coherent spin precession that is an essential factor, which, together with spin-dependent and spin-independent scattering rates, determines spin lifetimes in semiconductors and their heterostructures [2]. These spin-related effects have been studied extensively both theoretically and experimentally, yet there are still

*corresponding author; e-mail: skiera@fuw.edu.pl 
many open questions concerning the microscopic origin of the spin-splitting of the energy bands, their dependence on material combinations, on quantum well widths, or external factors like strain and/or electric field [2]. In this paper we attempt to answer some of these questions by first-principles, relativistic local density calculations.

Zinc-blende heterostructures or superlattices grown along [001] or [111] direction are technologically important systems for various spintronic applications. In these systems, the most important role in spin dynamics is played by linear- $\boldsymbol{k}$ spin splitting terms, which cause a camel-back or Mexican-hat-type structure of the conduction band in the neighborhood of the $\Gamma$ point. In bulk III-V materials, there are no linear- $\boldsymbol{k}$ terms in the conduction band at the $\Gamma$ point. However, there are linear- $\boldsymbol{k}$ spin splitting terms in other points of the bulk Brillouin zone (BZ). For example, the linear- $\boldsymbol{k}$ spin splitting of the conduction band is observed in a direction perpendicular to the $\Delta$ and $\Lambda$ lines, including $X$ and $L$ points at the edge of the BZ. In heterostructures, bulk $\boldsymbol{k}$ points along the $\Delta$ and $\Lambda$ lines are folded onto the heterostructure $\Gamma$ point. Therefore, they contribute to the linear- $\boldsymbol{k}$ spin splitting in the heterostructures. In this paper, we present systematic study of the linear- $\boldsymbol{k}$ spin splitting through the bulk BZ of the typical III-V semiconductors by means of the $a b$ initio relativistic calculations. Since the heterostructures are usually strained, we investigate also the role of the biaxial strain in inducing linear- $\boldsymbol{k}$ spin splitting. These studies are based on first-principles, relativistic local density calculations that provide very accurate description of the valence band spin splitting $\Delta_{0}$ in III-V bulks [3, 4]. Owing to the nearly complete lack of experimental data concerning the linear- $\boldsymbol{k}$ spin splitting, the present calculations provide theoretical predictions for these quantities.

\section{Results and discussion}

\subsection{Phonon induced linear- $\boldsymbol{k}$ spin splitting in $\Gamma$ point}

As it was mentioned already in the introduction, there is no linear- $\boldsymbol{k}$ spin splitting in the conduction band of an unstrained bulk zinc-blende III-V semiconductor. However, the manifold of heavy and light bands exhibit a very tiny linear- $\boldsymbol{k}$ spin splitting described by the formula $\varepsilon= \pm C\left\{k^{2} \pm\left[3\left(k_{x}^{2} k_{y}^{2}+k_{y}^{2} k_{z}^{2}+k_{z}^{2} k_{x}^{2}\right)\right]^{1 / 2}\right\}^{1 / 2}$. For example, the present $a b$ initio calculations give $C=0.0049 \mathrm{eV} \AA$ for GaAs, in reasonable agreement with previous calculations $[5,6]$. This spin splitting originates from the higher $(d, f, \ldots)$ angular momentum components of the pseudopotential and, therefore, cannot be described by any empirical theory (tight-binding, $\boldsymbol{k} \cdot \boldsymbol{p}$ ) involving only $s p$ states. However, a much larger linear- $\boldsymbol{k}$ splitting in the conduction band can be induced by elastic or phonon deformations of the bulk crystal.

A tetragonal biaxial strain reduces the $T_{d}$ symmetry to $D_{2 d}$ and leads to a bulk inversion asymmetry (BIA) effect in the spin splitting of the conduction 
band that is first order in the strain. The spin splitting energy can be written as $\Delta E_{\text {spin }}=2 \alpha_{\text {BIA }} \sqrt{k_{x}^{2}+k_{y}^{2}}$, with the constant $\alpha_{\text {BIA }}$ given by the formula $\alpha_{\mathrm{BIA}}=$ $B\left|\varepsilon_{x x}-\varepsilon_{z z}\right|$, where $B$ is a constant (so-called acoustic phonon constant, very often indicated in the literature as $C_{4} / 2$ ), and $\varepsilon_{x x}$ and $\varepsilon_{z z}$ are two components of the strain tensor. The calculated values of the acoustic phonon constant $B$ are equal to (in eV A) 0.002, 0.067, 0.126, 0.069, 0.200, 0.0385 for AlP, GaP, InP, AlAs, GaAs, and AlSb, respectively. The magnitude of the acoustic phonon constant is proportional to the strength of the spin-orbit coupling of the cation and inversely proportional to the energy gap that determines the coupling of cation $s$-states with anion $p$-states.

A frozen zone-center optical phonon, characterized by a relative shift $a u / 4$ of the two sublattices, leads to a uniaxial $C_{2 v}$ symmetry and therefore induces so-called structure inversion asymmetry (SIA) term in the conduction band that is first order in $u$. In addition, a BIA term is induced that is second order in $u$. Thus, the optical one-phonon spin deformation potential is determined by a SIA coupling, whereas the two-phonon spin deformation potential is determined by a BIA term. Specifically, we find $\alpha_{\text {SIA }}=-4.12 u$ and $\alpha_{\mathrm{BIA}}=135.3 u^{2}$ (in eV $\AA$ ) for the conduction band of GaAs. Therefore, in the presence of both terms the spin splitting energy of the conduction band is given by the formula [7]:

$$
\Delta E_{\uparrow \downarrow}=2 k_{\|} \sqrt{\left(\alpha_{\mathrm{BIA}}^{2}+\alpha_{\mathrm{SIA}}^{2}\right)-2 \alpha_{\mathrm{BIA}} \alpha_{\mathrm{SIA}} \sin (2 \theta)}
$$

where the lateral wave vector perpendicular to the axis determined by the phonon deformation is given by $k_{x}=k_{\|} \cos \theta, k_{y}=k_{\|} \sin \theta$. This anisotropic linear- $\boldsymbol{k}$ spin splitting is characteristic feature of the asymmetric semiconductor heterostructures exhibiting $C_{2 v}$ symmetry.

In this subsection we have shown the existence of the linear- $\boldsymbol{k}$ spin splitting in the conduction band near $\Gamma$ point in the strained semiconductors. However, even larger linear- $\boldsymbol{k}$ spin splitting has been observed in other points of the BZ.

\subsection{Zero field linear- $\boldsymbol{k}$ spin splitting in $X$ and $L$ points}

The $X$ and $L$ points in the BZ are folded onto $\Gamma$ point for heterostructures grown along [001] and [111] directions, respectively. In $X$ and $L$ points, the energy bands are spin degenerated. However, in the plane perpendicular to the $X$ and $L$ points, one observes the linear- $\boldsymbol{k}$ spin splitting of the conduction band. The calculated constants $\alpha_{\text {BIA }}$ for typical semiconductors are depicted in Fig. 1 . They are two orders of magnitude larger than the $\alpha_{\text {BIA }}$ constants for the conduction band near the $\Gamma$ point of the strained bulks with typical strains of the order of few $\%$. Generally, the constants $\alpha_{\text {BIA }}$ are larger for the $L$ point than for the $X$ point, but the trend is not particularly strong. The BIA constants in $L$ and $X$ point are also weakly linearly dependent on the external strain. The performed calculations show that the strain induced changes of $\alpha_{\mathrm{BI}}$ for $X$ and $L$ points are typically of the same order than the changes of $\alpha_{\mathrm{BIA}}$ in $\Gamma$ point discussed above. In 

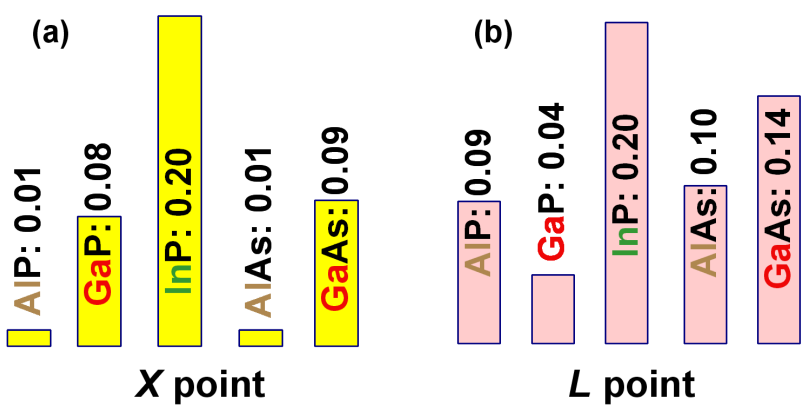

Fig. 1. Calculated values of the linear- $k$ spin splitting constant $\alpha_{\mathrm{BIA}}$ for AlP, GaP, InP, AlAs, and GaAs for (a) $X$ point and (b) $L$ point.

summary, the linear- $\boldsymbol{k}$ spin splitting in the $\Gamma$ point of heterostructures originates mostly from admixture of the wave functions of the bulk folded points to the wave function in $\Gamma$. The strain plays obviously only secondary role.

\subsection{Resonant linear- $\boldsymbol{k}$ spin splitting}

In the previous subsection, it has been shown that there is large linear- $\boldsymbol{k}$ spin splitting in the neighborhood of $X$ and $L$ points. Here, we demonstrate that the linear- $\boldsymbol{k}$ spin splitting can be resonantly enhanced in the certain regions of the BZ. As an example, we consider linear- $\boldsymbol{k}$ spin splitting for the first and second conduction bands of GaAs as calculated in the neighborhood of $\boldsymbol{k}$ points lying on the $\Delta$ line (let us note that for points lying strictly on the $\Delta$ line the band energies are spin degenerated). The spin splitting constant as a function of $k_{z} \in \Delta$ is depicted in Fig. 2. Owing to the crossing of the spin split bands (let us note that they transform according to different representations) the constant $\alpha_{\text {BIA }}$ changes the sign for $k_{z}$ approximately equal to (in units of $2 \pi / a$, with $a$ being the cubic lattice constant) 0.36 and 0.7 , for the second and the first band, respectively. It is clearly seen that the value of $\alpha_{\mathrm{BIA}}$ for both conduction bands reaches very high value of $0.3-0.44 \mathrm{eV} \AA$ for $k_{z}$ of order $0.3 \AA^{-1}$. This value of $k_{z}$ corresponds exactly to the wave vector at which the two conduction bands approach each other in an anticrossing manner, as is clearly demonstrated in the inset in Fig. 2. This behavior of $\alpha_{\text {BIA }}$ has universal character and is present in conduction as well as valence bands. For example, in the tensile strained GaAs, at $\Gamma$ point the light-hole band lies above the heavy-hole band. For a wave vector of the order of $0.1 \AA^{-1}$, the heavy-hole and light-hole bands anticross and the linear- $\boldsymbol{k}$ spin splitting shows resonant increase. The spin splitting (and constant $\left.\alpha_{\text {BIA }}\right)$ increases resonantly, whenever the two bands approach each other. This previously unnoticed phenomenon gives new possibilities to design spin splitting in the heterostructures. The spin splitting in the $\Gamma$ point of the heterostructure can be increased on purpose by the suitable choice of the quantum well width. On the other hand, one should avoid certain quantum well widths to guarantee 


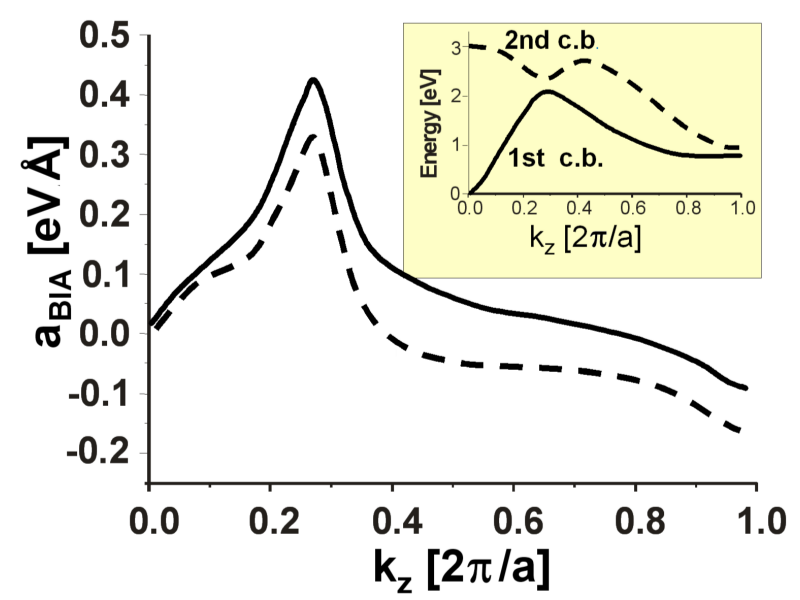

Fig. 2. Calculated values of the linear- $\boldsymbol{k}$ spin splitting constant $\alpha_{\mathrm{BIA}}$ for $k_{z}$ points along $\Delta$ axis for the first (solid line) and the second (dashed line) conduction bands of GaAs. The wave vectors $k_{z}$ are in the units of $2 \pi / a$, where $a$ indicates the cubic lattice constant. In these units, $X$ point corresponds to $k_{z}=1$. In the inset, the band energies of the first two conduction bands of GaAs are depicted.

the spin splitting as low as possible. The increase in the spin splitting for certain quantum well widths has been previously observed in theoretical calculations for AlAs/GaAs/AlAs systems [8].

\section{Conclusions}

The first-principles calculations clearly demonstrate that strain induced linear- $\boldsymbol{k}$ zero field spin splittings in III-V semiconductors are rather small. Most importantly, the calculations show that the $\boldsymbol{k}$-linear spin splitting can be resonantly enhanced when bands cross in a particular point of the Brillouin zone. This effect can be utilized in semiconductor heterostructures by choosing suitable well width and folding the $\boldsymbol{k}$ point onto heterostructure $\Gamma$ point. Thus, band structure engineering allows one to grossly enhance the spin splitting, which is favorable for applications where a fast spin precession is required.

\section{References}

[1] G.A. Prinz, Science 283, 330 (1999).

[2] I. Zutic, J. Fabian, S. Das Sarma, Rev. Mod. Phys. 76, 323 (2004).

[3] J.A. Majewski, M. Städele, P. Vogl, Mater. Res. Soc. Symp. Proc. 449, 887 (1997).

[4] J.A. Majewski, S. Birner, A. Trellakis, M. Sabathil, P. Vogl, Phys. Status Solidi C 1, 2003 (2004). 
[5] M.P. Surh, Ming-Fu Li, S.G. Louie, Phys. Rev. B 43, 4286 (1991).

[6] M. Cardona, N.E. Christensen, G. Fasol, Phys. Rev. Lett. 56, 2831 (1986).

[7] E.A. de Andrada e Silva, Phys. Rev. B 46, 1921 (1992).

[8] J.A. Majewski, P. Vogl, in: Physics of Semiconductors 2002, Proc. 26th Int. Conf. Phys. Semicond., Edinburgh 2002, Eds. A.R. Long, J.H. Davies, Institute of Physics Publishing, Bristol 2003, p. P305. 